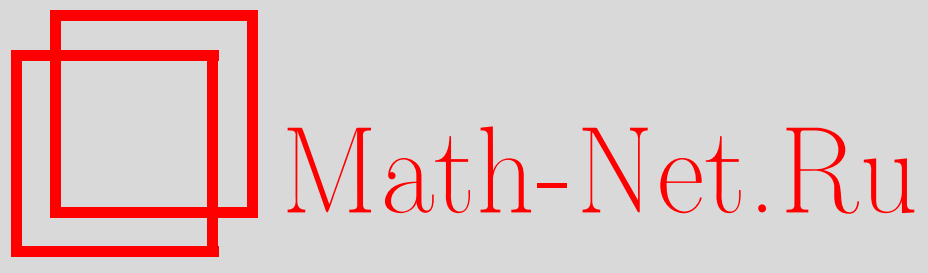

Д. И. Панюшев, О неприводимости коммутаторных многообразий, связанных с инволюциями простых алгебр Ли, Функи. анализ и его прил., 2004, том 38, выпуск 1, 47-55

DOI: https://doi.org/10.4213/faa95

Использование Общероссийского математического портала MathNet.Ru подразумевает, что вы прочитали и согласны с пользовательским соглашением

http://www . mathnet.ru/rus/agreement

Параметры загрузки:

IP: 54.84 .234 .179

26 апреля 2023 г., 18:35:11

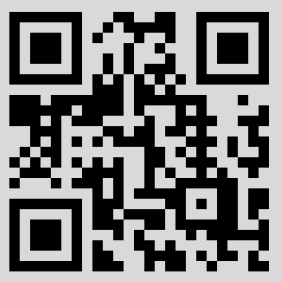


Функииональньй анализ и его приложения

2004, т. 38 , вып. 1, с. $47-55$

УДК 512.745

\title{
О неприводимости коммутаторных многообразий, связанных с инволюциями простых алгебр Ли*
}

\author{
(C) 2004. Д. И. ПАНЮШЕВ
}

\section{Введение}

Основное поле $\mathbb{k}$ алгебраически замкнуто и имеет характеристику нуль. Пусть $G$ - редуктивная алгебраическая группа и $\mathfrak{g}-$ ее алгебра Ли. В данной работе мы рассматриваем коммутаторные многообразия для представлений изотропии симметрических пространств. А именно, если $\mathfrak{g}=\mathfrak{g}_{0} \oplus \mathfrak{g}_{1}$ произвольная $\mathbb{Z}_{2}$-градуировка, то нас интересует многообразие $\mathfrak{C}_{1}=\{(x, y) \mid$ $[x, y]=0\} \subset \mathfrak{g}_{1} \times \mathfrak{g}_{1}$. В моей статье [6] было доказано, что $\mathfrak{C}_{1}$ неприводимо, если $\mathbb{Z}_{2}$-градуировка имеет максимальный ранг, т. е. $\mathfrak{g}_{1}$ содержит картановскую подалгебру из $\mathfrak{g}$. Здесь мы покажем, что $\mathfrak{C}_{1}$ неприводимо для $\left(\mathfrak{g}, \mathfrak{g}_{0}\right)=\left(\mathfrak{s l}_{2 n}, \mathfrak{s p}_{2 n}\right)$ или $\left(\mathbf{E}_{6}, \mathbf{F}_{4}\right)$. В случае симметрических пар ранга 1 будет показано, что число неприводимых компонент многообразия $\mathfrak{C}_{1}$ равно числу ненулевых нильпотентных $G_{0}$-орбит в $\mathfrak{g}_{1}$ немаксимальной размерности. Мы также обсудим общую задачу о неприводимости коммутаторных многообразий.

\section{$\S 1$. Напоминания о симметрических парах}

Пусть $\mathfrak{g}$ - редуктивная алгебраическая алгебра Ли и $\vartheta$ - ее инволютивный автоморфизм. Собственные подпространства автоморфизма $\vartheta$ задают в $\mathfrak{g}$ $\mathbb{Z}_{2}$-градуировку:

$$
\mathfrak{g}=\mathfrak{g}_{0} \oplus \mathfrak{g}_{1}, \quad \text { где } \mathfrak{g}_{i}=\left\{x \in \mathfrak{g} \mid \vartheta(x)=(-1)^{i} x\right\} .
$$

В этом случае мы также говорим, что $\left(\mathfrak{g}, \mathfrak{g}_{0}\right)-$ симметрическая пара. Если $G_{0}$ - связная алгебраическая группа с алгеброй Ли $\mathfrak{g}_{0}$, то $\mathfrak{g}_{1}$ - это $G_{0}$-модуль. Централизатор в $\mathfrak{g}$ произвольного подмножества $M \subset \mathfrak{g}$ обозначается через $\mathfrak{z}(M)$. Если $M \subset \mathfrak{g}_{1}$, то $\mathfrak{z}(M) \vartheta$-инвариантен и наследует $\mathbb{Z}_{2}$-градуировку от $\mathfrak{g}$. В частности, если элемент $s \in \mathfrak{g}_{1}$ полупрост, то $\left(\mathfrak{z}(s), \mathfrak{z}(s)_{0}\right)$ - это некоторая новая симметрическая пара, которая называется подсимметрической парой для $\left(\mathfrak{g}, \mathfrak{g}_{0}\right)$ или для $\vartheta$. Пусть $\mathfrak{c} \subset \mathfrak{g}_{1}-$ какое-либо максимальное подпространство, состоящее из коммутирующих полупростых элементов. Оно называется картановским. Все такие подпространства $G_{0}$-сопряжены. В следующем утверждении собраны другие полезные результаты из теории орбит и инвариантов группы $G_{0}$ в пространстве $\mathfrak{g}_{1}$.

ПреДЛОЖение 1.1 (Костант-Раллис [3]). Пусть $x \in \mathfrak{g}_{1}$.

(1) Орбита $G_{0} \cdot x$ замкнута $\Longleftrightarrow G_{0} \cdot x \cap \mathfrak{c} \neq \varnothing$; замыкание орбиты $G_{0} \cdot x$ содержит $0 \in \mathfrak{g}_{1} \Longleftrightarrow x-$ нильпотентный элемент.

(2) $\operatorname{dim} G \cdot x=2 \operatorname{dim} G_{0} \cdot x$.

*Работа выполнена при поддержке грантов РФФИ 01-01-00756 и 02-01-01041. 
(3) $G_{0} \cdot \mathfrak{c}$ плотно в $\mathfrak{g}_{1} u \mathfrak{z}(\mathfrak{c})_{1}=\mathfrak{c}$.

(4) $\mathfrak{g}_{1} / / G_{0}=\operatorname{Spec} \mathbb{k}\left[\mathfrak{g}_{1}\right]^{G_{0}}$ - аффинное пространство размерности $\operatorname{dim} \mathfrak{c} u$ морфизм факторизачии $\pi: \mathfrak{g}_{1} \rightarrow \mathfrak{g}_{1} / / G_{0}$ равноразмерен; каждый слой морфизма $\pi$ содержит конечное число $G_{0}$-орбит.

Из утверждения (2) предложения 1.1 следует, что $\operatorname{dim} \mathfrak{g}_{0}-\operatorname{dim} \mathfrak{z}(x)_{0}=\operatorname{dim} \mathfrak{g}_{1}-$ $\operatorname{dim} \mathfrak{z}(x)_{1}$ для любого $x \in \mathfrak{g}_{1}$.

ОПРеДЕЛЕНИЕ 1.2 . Пусть $x \in \mathfrak{g}_{1}$. Тогда $x$ называется $\vartheta$-регулярным, если его $G_{0}$-орбита имеет максимальную размерность; нильпотентный элемент $x$ называется $\vartheta$-отмеченным, если $\mathfrak{z}(x)_{1}$ не содержит нецентральных полупростых элементов.

Из утверждения (4) предложения 1.1 следует, что число $\vartheta$-регулярных нильпотентных орбит равно числу неприводимых компонент нуль-конуса $\mathcal{N}_{1}=$ $\pi^{-1}(\pi(0))$. Следующее утверждение известно, однако для удобства читателя мы приведем его короткое (новое) доказательство.

ЛЕмма 1.3. Любой $\vartheta$-регулярный нильпотентный элемент является $\vartheta$-отмеченным.

ДокаЗАТЕЛЬСтво. Допустим, что $x \in \mathcal{N}_{1}$ не является $\vartheta$-отмеченным, и пусть $s \in \mathfrak{z}(x)_{1}$ - нецентральный полупростой элемент. Тогда $a=x+s-$ это разложение Жордана для $a \in \mathfrak{g}_{1}$, и $2 \operatorname{dim} G_{0} \cdot(x+s)=\operatorname{dim} G \cdot(x+s)>\operatorname{dim} G \cdot x=$ $2 \operatorname{dim} G_{0} \cdot x$. Это значит, что размерность $G_{0}$-орбиты точки $x$ немаксимальна, т. е. $x$ не является $\vartheta$-регулярным.

\section{§2. Коммутаторные многообразия}

Напомним, что коммутаторное многообразие $\mathfrak{C}$ в $\mathfrak{g}$ - это множество пар коммутирующих элементов, $\mathfrak{C}=\{(x, y) \in \mathfrak{g} \times \mathfrak{g} \mid[x, y]=0\}$. Аналогично,

$$
\mathfrak{C}_{1}=\left\{(x, y) \in \mathfrak{g}_{1} \times \mathfrak{g}_{1} \mid[x, y]=0\right\}
$$

- это коммутаторное многообразие, ассоциированное с симметрической парой $\left(\mathfrak{g}, \mathfrak{g}_{0}\right)$ (или с $\left.\mathfrak{g}_{1}\right)$. Если требуется подчеркнуть зависимость коммутаторных многообразий от $\mathfrak{g}$, то мы пишем $\mathfrak{C}(\mathfrak{g})$ и $\mathfrak{C}\left(\mathfrak{g}_{1}\right)$ соответственно.

Фундаментальный результат Ричардсона [9] утверждает, что $\mathfrak{C}$ неприводимо и, более точно, $\mathfrak{C}=\overline{G \cdot(\mathfrak{t} \times \mathfrak{t})}$, где $\mathfrak{t}-$ произвольная картановская подалгебра в $\mathfrak{g}$. Однако до сих пор неизвестно, является ли $\mathfrak{C}$ нормальным и порождается ли идеал подмногообразия $\mathfrak{C}$ квадриками. Ситуация для симметрических пар сложнее и интереснее. С одной стороны, для некоторых $\vartheta$ удается доказать больше; с другой стороны, $\mathfrak{C}_{1}$ иногда бывает приводимым, а в некоторых случаях вопрос о неприводимости является открытым. Эффективный метод изучения коммутаторных многообразий, восходящий к Ричардсону [9], состоит в использовании разложения Жордана, индукции по $\operatorname{dim} \mathfrak{c}$ (переход к подсимметрическим парам) и рассмотрении проекции $p: \mathfrak{C}_{1} \rightarrow \mathfrak{g}_{1}$ коммутаторного многообразия на первый сомножитель. В работе [6] мы уже применяли этот метод к симметрическим парам и, более общо, к коммутаторным многообразиям, связанным с произвольными $\vartheta$-группами Э. Б. Винберга.

Число $\operatorname{dim} \mathfrak{c}$, где $\mathfrak{c} \subset \mathfrak{g}_{1}-$ картановское подпространство, называется рангом симметрической пары. В [6] было доказано, что $\mathfrak{C}_{1}=\overline{G_{0} \cdot(\mathfrak{c} \times \mathfrak{c})}$, если $\vartheta-$ инволюция максимального ранга (=инволюция Г. Вейля), причем $\mathfrak{C}_{1}-$ нормальное полное пересечение и его идеал в $\mathbb{k}\left[\mathfrak{g}_{1} \times \mathfrak{g}_{1}\right]$ порождается квадриками. С другой 
стороны, в $[6,3.5]$ приводится пример инволюции в $\mathfrak{g}=\mathfrak{s l}_{n}$, для которой $\mathfrak{C}_{1}$ имеет три неприводимых компоненты разных размерностей.

Ниже мы собираемся предъявить новые симметрические пары, для которых $\mathfrak{C}_{1}$ неприводимо, а также полностью разобрать случай симметрических пар ранга 1. Начнем с положительного результата, обеспечивающего неприводимость $\mathfrak{C}_{1}$.

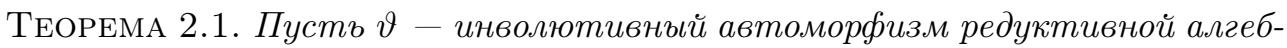
раической алгебры Ли $\mathfrak{g} u\left(\mathfrak{g}, \mathfrak{g}_{0}\right)$ - соответствующая симметрическая пара. Пусть для самой этой парь и для любой ее подсимметрической пары выполнено условие, что любой $\vartheta$-отмеченный нильпотентный элемент является $\vartheta$-регулярным. Тогда коммутаторное многообразие $\mathfrak{C}_{1} \subset \mathfrak{g}_{1} \times \mathfrak{g}_{1}$ неприводимо.

ДокАЗАтЕльство. Рассмотрим неприводимое подмногообразие

$$
\mathfrak{C}_{1}^{\mathfrak{c}}:=\overline{G_{0} \cdot(\mathfrak{c} \times \mathfrak{c})} \subset \mathfrak{C}_{1}
$$

Легко видеть (ср. [6, Sec. 3]), что

$$
\begin{aligned}
\operatorname{dim} \mathfrak{C}_{1}^{\mathfrak{c}} & =\operatorname{dim} G_{0}+2 \operatorname{dim} \mathfrak{c}-\operatorname{dim} \mathfrak{z}(\mathfrak{c})_{0} \\
& =\operatorname{dim} \mathfrak{g}_{1}+2 \operatorname{dim} \mathfrak{c}-\operatorname{dim} \mathfrak{z}(\mathfrak{c})_{1}=\operatorname{dim} \mathfrak{g}_{1}+\operatorname{dim} \mathfrak{c}
\end{aligned}
$$

Здесь используется тот факт, что $\mathfrak{z}(\mathfrak{c})_{1}=\mathfrak{c}$ и для точки общего положения $x \in \mathfrak{c}$ мы имеем $\mathfrak{z}(x)=\mathfrak{z}(\mathfrak{c})$. Как показано в $[6,3.7], \mathfrak{C}_{1}^{\mathfrak{c}}-$ неприводимая компонента многообразия $\mathfrak{C}_{1}$. Будем называть ее стандартной. Все остальные компоненты (если они есть) назовем странными. Покажем, что странных компонент быть не может. Обозначим через $\hat{p}$ ограничение проекции $p$ на $\mathfrak{C}_{1}^{\mathfrak{c}}$. Так как $\overline{G \cdot \mathfrak{c}}=\mathfrak{g}_{1}$, то проекция $\hat{p}$ сюръективна. Поэтому $\operatorname{dim} \hat{p}^{-1}(x) \geqslant \operatorname{dim} \mathfrak{c}$ для любого $x \in \mathfrak{g}_{1}$. $\mathrm{C}$ другой стороны, $\hat{p}^{-1}(x) \subset p^{-1}(x)=\{x\} \times \mathfrak{z}(x)_{1}$. Заметим, что $\operatorname{dim} \mathfrak{z}(x)_{1} \geqslant$ $\operatorname{dim} \mathfrak{c}$ для любого $x \in \mathfrak{g}_{1}$, причем равенство как раз характеризует $\vartheta$-регулярные элементы. Для $\vartheta$-регулярного нильпотентного элемента $e \in \mathfrak{g}_{1}$ это нам дает, что $p^{-1}(e)=\hat{p}^{-1}(e) \subset \mathfrak{C}_{1}^{\mathfrak{c}}$. Следовательно, $G_{0}$-орбита $\vartheta$-регулярного нильпотентного элемента $e \in \mathfrak{g}_{1}$ никогда не порождает странной компоненты многообразия $\mathfrak{C}_{1}$ (в том смысле, что $\left.G_{0} \cdot\left(\{e\} \times \mathfrak{z}(e)_{1}\right) \subset \mathfrak{C}_{1}^{\mathfrak{c}}\right)$. Иначе говоря, если $(x, y) \in \mathfrak{C}_{1}$ и $x-$ это $\vartheta$-регулярный нильпотентный элемент, то $(x, y) \in \mathfrak{C}_{1}^{\mathfrak{c}}$.

Число $\operatorname{dim}(\mathfrak{c} \cap[\mathfrak{g}, \mathfrak{g}])$ будем называть полупростым рангом симметрической пары. Копируя рассуждения Ричардсона, покажем индукцией по полупростому рангу, что $\mathfrak{C}_{1}=\mathfrak{C}_{1}^{\mathfrak{c}}$. Пусть $(x, y) \in \mathfrak{C}_{1}$ и $x=x_{s}+x_{n}$ - разложение Жордана.

1) Если $x_{s} \neq 0$ и $x_{s} \notin \mathfrak{z}(\mathfrak{g})$, то рассмотрим подсимметрическую пару $\left(\mathfrak{z}\left(x_{s}\right), \mathfrak{z}\left(x_{s}\right)_{0}\right)$. Ясно, что $x_{n}, y \in \mathfrak{z}\left(x_{s}\right)_{1}$ и даже $\left(x_{n}, y\right) \in \mathfrak{C}\left(\mathfrak{z}\left(x_{s}\right)_{1}\right)$. Не теряя общности, можно считать, что $x_{s} \in \mathfrak{c}$. Тогда $\mathfrak{c}$ является картановским подпространством и в $\mathfrak{z}\left(x_{s}\right)_{1}$. Однако полупростой ранг подсимметрической пары $\left(\mathfrak{z}\left(x_{s}\right), \mathfrak{z}\left(x_{s}\right)_{0}\right)$ меньше, чем ранг пары $\left(\mathfrak{g}, \mathfrak{g}_{0}\right)$. По предположению индукции $\left(x_{n}, y\right) \in \overline{Z\left(x_{s}\right)_{0} \cdot(\mathfrak{c} \times \mathfrak{c})}$. Но тогда и $\left(x_{s}+x_{n}, y\right) \in \overline{Z\left(x_{s}\right)_{0} \cdot(\mathfrak{c} \times \mathfrak{c})} \subset \mathfrak{C}_{1}^{\mathfrak{c}}$.

2) Если $x_{s} \in \mathfrak{z}(\mathfrak{g})$, то задача сводится к рассмотрению случая $x=x_{n}$.

3) Пусть $x=x_{n}$. Если $\mathfrak{z}\left(x_{n}\right)_{1}$ содержит нецентральный полупростой элемент, скажем $s$, то $c s+x_{n} \notin \mathcal{N}_{1}$ при $c \neq 0$ и $\left(c s+x_{n}, y\right) \in \mathfrak{C}_{1}$ при всех $c \in \mathbb{k}$. Как следует из п. 1$),\left(c s+x_{n}, y\right) \in \mathfrak{C}_{1}^{\mathfrak{c}}$ при $c \neq 0$. Следовательно, $\left(x_{n}, y\right) \in \mathfrak{C}_{1}^{\mathfrak{c}}$.

4) Осталось только рассмотреть случай, когда $x=x_{n}$ является $\vartheta$-отмеченным нильпотентным элементом. Но тогда, ввиду предположения теоремы, $x$ будет 
$\vartheta$-регулярным. Однако выше мы уже показали, что все пары коммутирующих элементов, включающие $\vartheta$-регулярные нильпотентные элементы, лежат B $\mathfrak{C}_{1}^{\mathfrak{c}}$.

ЗАмЕчАниЕ. В сущности, индуктивные рассуждения ’̀ la Ричардсон показывают, что приводимость многообразия $\mathfrak{C}_{1}$ всегда связана с тем, что для некоторой подсимметрической пары $\left(\overline{\mathfrak{g}}, \overline{\mathfrak{g}}_{0}\right)$ в $\left(\mathfrak{g}, \mathfrak{g}_{0}\right)$ и для некоторого $\vartheta$-отмеченного нильпотентного элемента $\bar{e} \in \overline{\mathfrak{g}}_{1}$ окажется, что $\bar{G}_{0} \cdot\left(\{\bar{e}\} \times\left(\mathfrak{z}(\bar{e}) \cap \overline{\mathfrak{g}}_{1}\right)\right)$ не лежит в стандартной компоненте многообразия $\mathfrak{C}\left(\overline{\mathfrak{g}}_{1}\right)$.

\section{§3. Новые примеры неприводимых коммутаторных многообразий}

Симметрическая пара $\left(\mathfrak{s l}_{\mathbf{2 n}}, \mathfrak{s p}_{2 n}\right)$. Наша цель - доказать, что в этом случае применима теорема 2.1 и, следовательно, $\mathfrak{C}_{1}$ неприводимо. Начнем с явной матричной модели $\mathbb{Z}_{2}$-градуировки в $\mathfrak{g}=\mathfrak{s l}_{2 n}$. Пусть $\Upsilon_{n}$ есть $n \times n$ матрица с единицами вдоль побочной диагонали и нулями на остальных местах. Положим $J=\left(\begin{array}{cc}0 & \Upsilon_{n} \\ -\Upsilon_{n} & 0\end{array}\right)$ и $\vartheta(X)=J X^{t} J$. Тогда $\mathfrak{s p}_{2 n} \simeq \mathfrak{g}_{0}=\left\{X \in \operatorname{Mat}_{2 n}(\mathbb{k}) \mid\right.$ $X J$ симметрична $\}$ и $\mathfrak{g}_{1}=\left\{X \in \operatorname{Mat}_{2 n}(\mathbb{k}) \mid X J\right.$ кососимметрична $\}$. Для $A \in$ $\operatorname{Mat}_{n}(\mathbb{k})$ положим $\hat{A}=\Upsilon_{n} A^{t} \Upsilon_{n}$. Легко видеть, что преобразование $A \mapsto \hat{A}-$ это транспонирование относительно побочной диагонали. В этих обозначениях мы имеем

$$
\begin{aligned}
& \mathfrak{g}_{0}=\left\{\left(\begin{array}{cc}
A & B \\
C & -\hat{A}
\end{array}\right) \mid \hat{B}=B, \widehat{C}=C\right\}, \\
& \mathfrak{g}_{1}=\left\{\left(\begin{array}{ll}
N & P \\
Q & \widehat{N}
\end{array}\right) \mid \hat{P}=-P, \widehat{Q}=-Q, \operatorname{tr} N=0\right\} .
\end{aligned}
$$

ПРЕДЛОЖЕНИЕ 3.1. (i) Пусть $e \in \mathfrak{g}_{1}$ - нилъпотентный элемент. Тогда $S L_{2 n} \cdot e \cap \mathfrak{g}_{1}$ - это единственная $S p_{2 n}$-орбита.

(ii) Если $\mathcal{O}$ - произвольная нильпотентная $S L_{2 n}$-орбита, то $\mathcal{O} \cap \mathfrak{g}_{1} \neq \varnothing$ тогда и только тогда, когда любая часть разбиения, соответствующего $\mathcal{O}$, встречается четное число раз.

ДокАЗАтЕльство. (i) Это утверждение хорошо известно. Чисто классификационное доказательство можно найти в [5]. Более концептуальное рассуждение имеется в $[8,4.3]$.

(ii) Инволюция $\vartheta$, выделяющая $\mathfrak{s p}_{2 n}$, имеет следующую диаграмму Сатаке:

( $n$ черных кружков и $n-1$ белых). Как отмечено в [7, p. 1469], результат Антоняна [1, теорема 1] показывает, что нильпотентная $G$-орбита пересекает $\mathfrak{g}_{1}$ тогда и только тогда, когда ее характеристика имеет нулевые отметки на черных кружках диаграммы Сатаке и равные отметки на белых кружках, соединенных стрелками, если последние имеются. В данной ситуации это как раз означает, что все части соответствующего разбиения встречаются четное число раз. (Следует воспользоваться алгоритмом написания характеристики по разбиению, см. [11, IV.1.13].)

Далее нам потребуются подалгебра $\mathfrak{s}=\{B=C=0\} \subset \mathfrak{g}_{0}$ и подпространство $V=\{P=Q=0\} \subset \mathfrak{g}_{1}$. Очевидно, что $\mathfrak{s} \simeq \mathfrak{g l}_{n}, V \simeq \mathfrak{s l}_{n}$ и по модулю центра 
представление алгебры $\mathfrak{s}$ на $V$ изоморфно присоединенному представлению алгебры $\mathfrak{s l}_{n}$.

Теорема 3.2. (i) Имеются естественные биекиии между множествами

- нильпотентных $S L_{2 n}$-орбит в $\mathfrak{g}=\mathfrak{s l}_{2 n}$, пересекающих $\mathfrak{g}_{1}$,

- нильпотентных $G_{0}$-орбит в $\mathfrak{g}_{1}$,

- нильпотентных $\mathfrak{s - о р б и т ~ в ~} V$.

Эти биекиии задаются отображениями

$$
\mathcal{O} \mapsto \mathcal{O} \cap \mathfrak{g}_{1} \mapsto \mathcal{O} \cap V
$$

где $\mathcal{O}$ - нильпотентная орбита в $\mathfrak{g}$. При этом, если $\mathcal{O}$ задается разбиением $\left(n_{1}^{2 k_{1}}, \ldots, n_{t}^{2 k_{t}}\right)$, то $\mathcal{O} \cap V$ дается разбиением $\left(n_{1}^{k_{1}}, \ldots, n_{t}^{k_{t}}\right)$;

(ii) Биекиии из n. (i) уваэсают примыкания орбит, m. е.

$$
\overline{\mathcal{O}} \supset \mathcal{O}^{\prime} \Longleftrightarrow \overline{\mathcal{O} \cap \mathfrak{g}_{1}} \supset \mathcal{O}^{\prime} \cap \mathfrak{g}_{1} \Longleftrightarrow \overline{\mathcal{O} \cap V} \supset \mathcal{O}^{\prime} \cap V .
$$

(iii) $B \mathfrak{g}_{1}$ есть единственная $\vartheta$-регулярная нильпотентная орбита, и любой $\vartheta$-отмеченный нильпотентный элемент в $\mathfrak{g}_{1}$ является регулярным;

(iv) Любая подсимметрическая пара в $\left(\mathfrak{s l}_{2 n}, \mathfrak{s p}_{2 n}\right)$ есть прямая сумма пар такого же вида и тривиальной парь.

Доказательство. (i) Обозначим через $\mathcal{M}^{(2 n)}, \mathcal{M}^{\prime}$ и $\mathcal{M}^{(n)}$ три множества нильпотентных орбит, фигурирующих в условии. О биекции между первыми двумя говорится в предложении 3.1(i). Имеется также естественное отображение $\nu: \mathcal{M}^{(n)} \rightarrow \mathcal{M}^{(2 n)}$, при котором $n \times n$-матрица $N$ переходит в $\left(\begin{array}{cc}N & 0 \\ 0 & \widehat{N}\end{array}\right) \in V$. Очевидно, это отображение удваивает разбиение (т. е. количество жордановых блоков каждого размера). Поэтому, согласно предложению 3.1(ii), $\nu$ - биекция.

(ii) Обе импликации «ऍ» очевидны. Поэтому достаточно установить, что из $\overline{\mathcal{O}} \supset \mathcal{O}^{\prime}$ следует $\overline{\mathcal{O} \cap V} \supset \mathcal{O}^{\prime} \cap V$. А это, в свою очередь, следует из того, что примыкание нильпотентных орбит определятся стандартным частичным порядком на множестве разбиений $[2,6.2]$ и связью между разбиениями, задающими орбиты $\mathcal{O}$ и $\mathcal{O} \cap V$.

(iii) Предыдущие биекции показывают, что единственная $\vartheta$-регулярная орбита в $\mathfrak{g}_{1}$ происходит из нильпотентной орбиты в $\mathfrak{s l}_{2 n}$ с разбиением $(n, n)$, иначе говоря, из регулярной нильпотентной $S L_{n}$-орбиты в $V$. Любая же другая орбита происходит из нерегулярной $S L_{n}$-орбиты. Централизатор нерегулярного нильпотентного элемента в $\mathfrak{s l}_{n}$ всегда содержит полупростые элементы. При отождествлении $\mathfrak{s l}_{n}$ с пространством $V$ (посредством «удвоения», как в п. (i)) это дает нам полупростой элемент в $\mathfrak{z}(e)_{1}$ для любого $e \in V \subset \mathfrak{g}_{1}$, не являющегося $\vartheta$-регулярным.

(iv) Подпространство $\mathfrak{c}=\left\{\operatorname{diag}\left(t_{1}, \ldots, t_{n}, t_{n}, \ldots, t_{1}\right) \mid \sum t_{i}=0\right\} \subset V$ является картановским в $\mathfrak{g}_{1}$. Ввиду сопряженности картановских подпространств достаточно рассматривать централизаторы элементов из с. В этом случае структура централизатора $\mathfrak{z}(x), x \in \mathfrak{c}$, видна непосредственно из матричного описания алгебр $\mathfrak{g}_{0}$ и $\mathfrak{g}_{1}$.

СЛЕДСТВИЕ 3.3. Для $\left(\mathfrak{s l}_{2 n}, \mathfrak{s p}_{2 n}\right)$ коммутаторное многообразие $\mathfrak{C}_{1}$ неприводимо.

ДокАЗАтЕЛЬство. Это следует из теоремы 2.1 и теоремы 3.2(iii)-(iv). 
Симметрическая пара $\left(\mathbf{E}_{\mathbf{6}}, \mathbf{F}_{\mathbf{4}}\right)$. Будем следовать тем же путем, что и выше. Сначала немного нумерологии. В данном случае $\operatorname{dim} \mathfrak{g}_{0}=52, \operatorname{dim} \mathfrak{g}_{1}=26$, $\operatorname{dim} \mathfrak{c}=2$. Диаграмма Сатаке данной пары - это

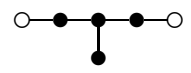

В $\mathbf{E}_{6}$ есть только две ненулевые нильпотентные орбиты с характеристиками, имеющими нулевые отметки на черных кружках:

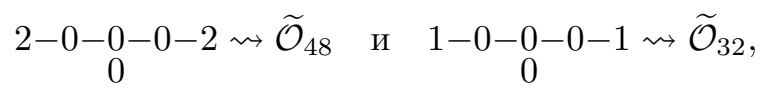

где индекс обозначает размерность. Так как инволюция $\vartheta$ связана с диаграммным автоморфизмом алгебры $\mathbf{E}_{6}$, то ввиду $[8,4.3]$ пересечения $\tilde{\mathcal{O}}_{i} \cap \mathfrak{g}_{1}-$ это в точности $\mathbf{F}_{4}$-орбиты (половинной размерности). Таким образом, $\mathfrak{g}_{1}$ имеет две ненулевые нильпотентные $\mathbf{F}_{4}$-орбиты: $\mathcal{O}_{24}$ и $\mathcal{O}_{16}$. Очевидно, что $\mathcal{O}_{24}$ - это $\vartheta$-регулярная орбита. Что касается $\mathcal{O}_{16}$ (или $\tilde{\mathcal{O}}_{32}$ ), то мы сравним редуктивные части централизаторов $\mathfrak{z}(e)$ и $\mathfrak{z}(e)_{0}$ для $e \in \mathcal{O}_{16}$. По таблицам Элашвили [12, табл. 4] находим, что $\mathfrak{z}(e)^{\mathrm{red}}=\mathbf{B}_{3} \times T_{1}$. Здесь $T_{1}-$ это алгебра Ли одномерного тора. С другой стороны, $e-$ старший вектор в 26-мерном неприводимом представлении алгебры $\mathbf{F}_{4}$. Поэтому $\left(\mathfrak{z}(e)_{0}\right)^{\text {red }}=\mathbf{B}_{3}$. Следовательно, $\mathfrak{z}(e)_{1} \supset T_{1}$ и $е$ не является $\vartheta$-отмеченным.

Единственная нетривиальная собственная подсимметрическая пара, связанная с $\left(\mathbf{E}_{6}, \mathbf{F}_{4}\right)$ - это $\left(\mathbf{D}_{5}, \mathbf{B}_{4}\right)$. У последней есть единственная нетривиальная нильпотентная орбита. Следовательно, здесь применима теорема 2.1, и мы получаем

ПРЕДЛОЖЕНИЕ 3.4. Для $\left(\mathbf{E}_{6}, \mathbf{F}_{4}\right)$ коммутаторное многообразие $\mathfrak{C}_{1}$ неприводимо.

\section{§4. Коммутаторные многообразия для симметрических пар ранга 1}

В этом параграфе мы рассматриваем симметрические пары, для которых $\operatorname{dim} \mathfrak{c}=1$. Поэтому стандартная неприводимая компонента $\mathfrak{C}_{1}^{\mathfrak{c}}$ имеет размерность $\operatorname{dim} \mathfrak{g}_{1}+1$. Напомним, что $\mathcal{N}_{1} \subset \mathfrak{g}_{1}-$ нуль-конус и что, как показано в доказательстве теоремы $2.1, \vartheta$-регулярная орбита из $\mathcal{N}_{1}$ никогда не порождает странной неприводимой компоненты.

Теорема 4.1. Любая нетривиальная $G_{0}$-орбита в $\mathfrak{g}_{1}$, не являющаяся $\vartheta$-регулярной, порождает странную неприводимую компоненту. Более точно, если $\operatorname{dim} G_{0} \cdot e<\operatorname{dim} \mathfrak{g}_{1}-1$, mо $\overline{G_{0} \cdot\left(\{e\} \times \mathfrak{z}(e)_{1}\right)}-$ странная неприводимая компонента многообразия $\mathfrak{C}_{1}$ размерности $\operatorname{dim} \mathfrak{g}_{1}$. При этом разные орбиты порождают разные неприводимые компоненты.

Доказательство. У нас $\operatorname{dim} \mathfrak{c}=1$; поэтому $\operatorname{dim} G_{0} \cdot x \leqslant \operatorname{dim} \mathfrak{g}_{1}-1$ для любого $x \in \mathfrak{g}_{1}$. Так как $\operatorname{dim} G_{0} \cdot x=\operatorname{dim} \mathfrak{g}_{0}-\operatorname{dim} \mathfrak{z}(x)_{0}=\operatorname{dim} \mathfrak{g}_{1}-\operatorname{dim} \mathfrak{z}(x)_{1}$, то

$$
x \text { является } \vartheta \text {-регулярным } \Longleftrightarrow \operatorname{dim} \mathfrak{z}(x)_{1}=1 \Longleftrightarrow \mathfrak{z}(x)_{1}=\mathbb{k} x
$$

В случае ранга 1 любой элемент в $\mathfrak{g}_{1}$ либо полупрост, либо нильпотентен, причем все полупростые элементы $\vartheta$-регулярны. Следовательно, нерегулярный элемент обязательно нильпотентен. Кроме того, $\mathfrak{C}_{1}^{\mathfrak{c}}-$ это просто множество пар пропорциональных элементов из $\mathfrak{g}_{1}$. 
Если $e \in \mathcal{N}_{1} \backslash\{0\}$ - нерегулярный элемент, то $\operatorname{dim} \mathfrak{z}(e)_{1} \geqslant 2$. Пусть $e^{\prime} \in$ $\mathfrak{z}(e)_{1} \backslash \mathbb{k} e$. Тогда $\left(e, e^{\prime}\right) \in \mathfrak{C}_{1} \backslash \mathfrak{C}_{1}^{\mathfrak{c}}$. Точнее, $\overline{G_{0} \cdot\left(\{e\} \times \mathfrak{z}(e)_{1}\right)}-$ это $\operatorname{dim} \mathfrak{g}_{1}$-мерное неприводимое подмногообразие в $\mathfrak{C}_{1}$, не лежащее в $\mathfrak{C}_{1}^{\mathfrak{c}}$. Заметим, что $G_{0} \cdot(\{x\} \times$ $\left.\mathfrak{z}(x)_{1}\right) \subset \mathfrak{C}_{1}^{\mathfrak{c}}$, если $x \quad \vartheta$-регулярен (это следует из $\left.(*)\right)$ или $x=0$. Поэтому

$$
\mathfrak{C}_{1}=\mathfrak{C}_{1}^{\mathfrak{c}} \cup\left(\bigcup_{i \in I} \overline{G_{0} \cdot\left(\left\{e_{i}\right\} \times \mathfrak{z}\left(e_{i}\right)_{1}\right)}\right),
$$

где $e_{i}$ пробегает множество представителей всех ненулевых $\vartheta$-нерегулярных $G_{0}$-орбит в $\mathfrak{g}_{1}$. Положим $\mathfrak{C}^{(i)}=\overline{G_{0} \cdot\left(\left\{e_{i}\right\} \times \mathfrak{z}\left(e_{i}\right)_{1}\right)}$. Тогда

- $I$ конечно ввиду конечности множества нильпотентных орбит, см. 1.1(4);

- $\operatorname{dim} \mathfrak{C}^{(i)}=\operatorname{dim} \mathfrak{g}_{1}$ для любого $i \in I$;

- $\mathfrak{C}^{(i)} \neq \mathfrak{C}^{(j)}$ (ибо они имеют разные проекции на первый сомножитель);

- $\mathfrak{C}^{(i)} \not \subset \mathfrak{C}_{1}^{\mathfrak{c}}$ (см. выше).

Значит, $(* *)$ - это разложение на неприводимые компоненты.

Пример. Следующая табличка содержит все симметрические пары ранга 1 и указывает для каждой число неприводимых компонент у $\mathfrak{C}_{1}$.

\begin{tabular}{|c|c|c|c|c|}
\hline $\begin{array}{l}\text { Симметриче- } \\
\text { ская пара }\end{array}$ & $\begin{array}{c}\left(\mathfrak{s o}_{n}, \mathfrak{s o}_{n-1}\right) \\
n \geqslant 3\end{array}$ & $\begin{array}{c}\left(\mathfrak{s p}_{2 n}, \mathfrak{s p}_{2 n-2} \times \mathfrak{s p}_{2}\right) \\
n \geqslant 2\end{array}$ & $\left(\mathbf{F}_{4}, \mathbf{B}_{4}\right)$ & $\begin{array}{c}\left(\mathfrak{s l}_{n}, \mathfrak{s l}_{n-1} \times T_{1}\right) \\
n \geqslant 3\end{array}$ \\
\hline$\# \operatorname{Irr}\left(\mathfrak{C}_{1}\right)$ & 1 & 2 & 2 & 3 \\
\hline
\end{tabular}

Последний столбец как раз представляет собой пример, рассмотренный в [6, 3.5].

ЗАмЕчАния. 1. В случае пар ранга 1 любой ненулевой нильпотентный элемент в $\mathfrak{g}_{1}$ является $\vartheta$-отмеченным (ибо все ненулевые полупростые элементы в $\mathfrak{g}_{1} \vartheta$-регулярны).

2. Вопрос о неприводимости коммутаторных многообразий для пар ранга 1 рассматривается в недавней работе [10]. Наши результаты были получены одновременно и независимо. Однако наш подход короче и дает явное описание неприводимых компонент.

\section{§5. Что дальше?}

Отбрасывая симметрические пары максимального ранга, два случая, рассмотренных в $\S 3$, и пары ранга 1, мы видим, что вопрос о неприводимости коммутаторного многообразия остается открытым для следующих симметрических пар:

$$
\begin{aligned}
& \left(\mathfrak{s l}_{n+m}, \mathfrak{s l}_{n} \times \mathfrak{s l}_{m} \times T_{1}\right), \quad 1<m \leqslant n, \quad\left(\mathfrak{s o}_{n+m}, \mathfrak{s o}_{n} \times \mathfrak{s o}_{m}\right), \quad m \geqslant 2, n-m \geqslant 2, \\
& \left(\mathfrak{s p}_{n+m}, \mathfrak{s p}_{n} \times \mathfrak{s p}_{m}\right), \quad 1<m \leqslant n, \quad\left(\mathfrak{s o}_{2 n}, \mathfrak{s l}_{n} \times T_{1}\right), \quad n \geqslant 4, \quad\left(\mathbf{E}_{6}, \mathbf{D}_{5} \times T_{1}\right),
\end{aligned}
$$

$$
\left(\mathbf{E}_{7}, \mathbf{E}_{6} \times T_{1}\right), \quad\left(\mathbf{E}_{6}, \mathbf{A}_{5} \times \mathbf{A}_{1}\right), \quad\left(\mathbf{E}_{7}, \mathbf{D}_{6} \times \mathbf{A}_{1}\right), \quad\left(\mathbf{E}_{8}, \mathbf{E}_{7} \times \mathbf{A}_{1}\right) .
$$

Можно проверить, что каждая из этих пар содержит $\vartheta$-отмеченные нильпотентные элементы, не являющиеся $\vartheta$-регулярными, так что теорема 2.1 здесь неприменима. Однако я полагаю, что $\mathfrak{C}_{1}$ неприводимо во всех этих случаях. (Для классических серий и малых значений параметров $m, n$ неприводимость можно проверять непосредственно.) Это может быть сформулировано и в более красивых и инвариантных терминах. 
Рассмотрим представление $\rho_{0}: G_{0} \rightarrow G L\left(\mathfrak{g}_{1}\right)$, ассоциированное с симметрической парой $\left(\mathfrak{g}, \mathfrak{g}_{0}\right)$. Пусть $\widetilde{G}_{0}-$ наибольшая связная подгруппа в $G L\left(\mathfrak{g}_{1}\right)$, такая, что $\mathbb{k}\left[\mathfrak{g}_{1}\right]^{G_{0}}=\mathbb{k}\left[\mathfrak{g}_{1}\right]^{\widetilde{G}_{0}}$.

ГиПотезА. Многообразие $\mathfrak{C}_{1}$ неприводимо тогда и толъко тогда, когда симметрическая пара $\left(\mathfrak{g}, \mathfrak{g}_{0}\right)$ удовлетворяет условию $G_{0}=\widetilde{G}_{0}$.

Все случаи, когда $G_{0} \neq \widetilde{G}_{0}$, были найдены в [4]. Оказывается (если $\mathfrak{g}$ простая), это в точности симметрические пары ранга 1 , отличные от $\left(\mathfrak{s o}_{n}, \mathfrak{s o}_{n-1}\right)$ ! Таким образом, теорема 4.1 и классификация, полученная в [4], доказывают необходимость условия в гипотезе 5.1. Отметим также, что эта гипотеза, в частности, утверждает, что если ранг неразложимой симметрической пары $\geqslant 2$, то $\mathfrak{C}_{1}$ неприводимо.

На эту ситуацию можно взглянуть и с более общей точки зрения. Забудем пока о симметрических парах и рассмотрим какое-нибудь представление $\rho: G \rightarrow G L(V)$ связной редуктивной группы $G$. Следуя работе [6], рассмотрим «коммутаторное» многообразие $\mathfrak{C}_{V} \subset V \times V^{*}$. По определению

$$
\mathfrak{C}_{V}=\left\{(v, \xi) \in V \times V^{*} \mid\langle\xi, \mathfrak{g} \cdot v\rangle=0\right\} .
$$

Здесь $\langle$,$\rangle обозначает естественное спаривание между V$ и $V^{*}$. Иначе говоря, если $\varphi: V \times V^{*} \rightarrow \mathfrak{g}^{*} \simeq \mathfrak{g}$ - отображение момента (см. [6, Sес. 1]), то $\mathfrak{C}_{V}=\varphi^{-1}(0)$. Напомним, что действие $(G: V)$ называется обозримым, если все слои орбитного морфизма $\pi_{G, V}: V \rightarrow V / / G$ содержат конечное число орбит; действие называется стабилъным, если среди $G$-орбит максимальной размерности найдется замкнутая. Как и выше, определим группу $\widetilde{G}$ как максимальную связную подгруппу в $G L(V)$, обладающую тем свойством, что $\mathbb{k}[V]^{G}=\mathbb{k}[V]^{\widetilde{G}}$. При этом не предполагается, что группа $\widetilde{G}$ обязательно редуктивна.

Tеорема 5.2. Пусть действие $(G: V)$ стабилъно и обозримо. Предположим что $\widetilde{G} \supsetneqq G$ и, более того, найдется точка $v \in V$, такая, что $\operatorname{dim} \widetilde{G} \cdot v>$ $\operatorname{dim} G \cdot v$. Тогда $\mathfrak{C}_{V}$ приводимо.

ДоказАтельство. Так как $G$ и $\widetilde{G}$ имеют одинаковые инварианты в $V$, то $V / / G \simeq V / / \widetilde{G}$ и слои орбитных морфизмов у них одинаковы. Ввиду стабильности действия группы $G$ слой общего положения является замкнутой $G$-орбитой и, следовательно, замкнутой $\widetilde{G}$-орбитой. Таким образом, действие $(\widetilde{G}: V)$ также стабильно и обозримо. Рассмотрим коммутативную диаграмму, включающую отображения момента для действий групп $G$ и $\widetilde{G}$ :

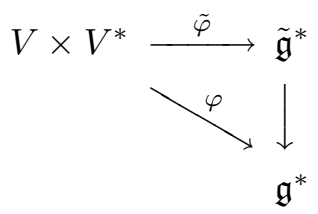

Здесь вертикальная стрелка - это отображение, двойственное к вложению $\mathfrak{g} \hookrightarrow \tilde{\mathfrak{g}}$. Положим $\widetilde{\mathfrak{C}}_{V}=\tilde{\varphi}^{-1}(0)$. Ясно, что $\mathfrak{C}_{V} \supset \widetilde{\mathfrak{C}}_{V}$. Согласно [6, 3.7], из обозримости и стабильности обоих рассматриваемых действий следует, что

$$
\operatorname{dim} \mathfrak{C}_{V}=\operatorname{dim} V+\operatorname{dim} V / / G=\operatorname{dim} \widetilde{\mathfrak{C}}_{V}
$$


(Соответствующее утверждение в [6] формулируется для редуктивных групп. Однако, по существу, редуктивность там нигде не используется.) Поэтому достаточно показать, что $\widetilde{\mathfrak{C}}_{V} \neq \mathfrak{C}_{V}$. По нашему предположению найдется точка $v \in V$, такая, что $\tilde{\mathfrak{g}} \cdot v \supsetneqq \mathfrak{g} \cdot v$. Выберем $\xi \in V^{*}$ так, чтобы $\langle\xi, \mathfrak{g} \cdot v\rangle=0$ и $\langle\xi, \tilde{\mathfrak{g}} \cdot v\rangle \neq 0$. Тогда $(v, \xi) \in \mathfrak{C}_{V} \backslash \widetilde{\mathfrak{C}}_{V}$.

Заметим, что может случиться так, что $\widetilde{G} \supsetneqq G$ и $\mathbb{k}[V]^{\widetilde{G}}=\mathbb{k}[V]^{G} \neq \mathbb{k}$, но все орбиты этих групп одинаковы! Например, пусть $G=\mathbf{G}_{2}$ и $V$ - пространство 7-мерного представления. Тогда $\widetilde{G}=S O_{7}$.

Возвращаясь теперь к симметрическим парам, мы видим, что теорема 5.2 частично объясняет ситуацию с симметрическими парами ранга 1. Если пара $\left(\mathfrak{g}, \mathfrak{g}_{0}\right)$ имеет ранг 1 и отлична от $\left(\mathfrak{s o}_{n}, \mathfrak{s o}_{n-1}\right)$, то $\widetilde{G}_{0}=S O\left(\mathfrak{g}_{1}\right) \neq G_{0}$. При этом любая $\vartheta$-нерегулярная $G_{0}$-орбита в $\mathfrak{g}_{1}$ порождает нильпотентную $S O\left(\mathfrak{g}_{1}\right)$-орбиту большей размерности. И в этом-то и состоит причина появления странных неприводимых компонент у $\mathfrak{C}\left(\mathfrak{g}_{1}\right)$.

Интересно было бы найти все обозримые и стабильные действия $(G: V)$, для которых $\widetilde{G} \neq G$. Возможно, таких просто не бывает, если $\operatorname{dim} V / / G>1$.

\section{ЛитерАТУРА}

1. Антонян Л. В. О классификации однородных элементов $\mathbb{Z}_{2}$-градуированных полупростых алгебр Ли. Вестник МГУ, сер. матем., мех., №2, 29-34 (1982).

2. Collingwood D. H., McGovern W. Nilpotent orbits in semisimple Lie algebras. Van Nostrand Reinhold Co., New York, 1993.

3. Kostant B., Rallis S. Orbits and representations associated with symmetric spaces. Amer. J. Math., 93, 753-809 (1971).

4. Levasseur T., Ushirobira $R$. Adjoint vector fields on the tangent space of semisimple symmetric spaces. J. Lie Theory, 9, 293-304 (1999).

5. Ohta T. Closures of nilpotent orbits in classical symmetric pairs and their singularities. Tohoku Math. J., 43 (2), 161-213 (1991).

6. Panyushev D. The Jacobian modules of a Lie algebra and geometry of commuting varieties. Compositio Math., 94, 181-199 (1994).

7. Panyushev D. On spherical nilpotent orbits and beyond. Ann. Inst. Fourier, 49, 14531476 (1999).

8. Reeder M. Desingularizations of some unstable orbit closures. Pacific J. Math., 167, 327-343 (1995).

9. Richardson $R$. W. Commuting varieties of semisimple Lie algebras and algebraic groups. Compositio Math., 38, 311-327 (1979).

10. Sabourin $H ., Y u R$. W. T. Sur l'irreductibilite de la variété commutante d'une paire symétrique réductive de rang 1. Bull. Sci. Math., 126, 143-150 (2002).

11. Спрингер T. А., Штейнберг Р. Классы сопряженных элементов, В кн.: Семинар по алгебраическим группам. Мир, М., 162-262, 1973.

12. Элашвили А. Г. Централизаторы нильпотентных элементов в полупростых алгебрах Ли. Труды Тбилисского матем. института им. Размадзе, 46, 109-132 (1975).

Независимый московский университет e-mail: panyush@mccme.ru
Поступило в редакцию 20 сентября 2002 г. 